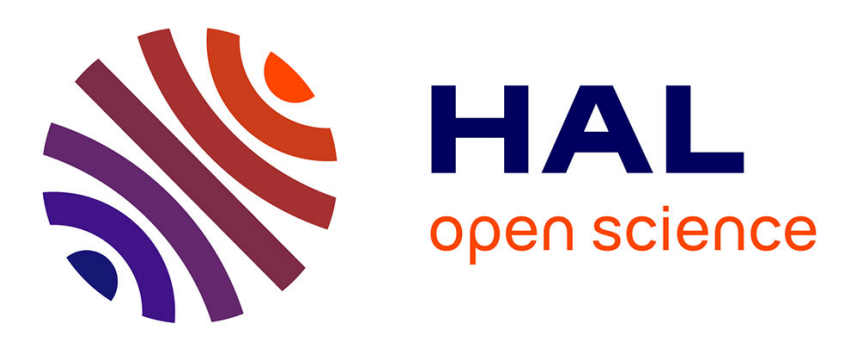

\title{
Optimal Location of a Mobile Sensor Continuum for Environmental Monitoring \\ Didier Georges
}

\section{To cite this version:}

Didier Georges. Optimal Location of a Mobile Sensor Continuum for Environmental Monitoring. CPDE 2013 - 1st IFAC Workshop on Control of Systems Modeled by Partial Differential Equations, Sep 2013, Paris, France. hal-00834645

\section{HAL Id: hal-00834645 \\ https://hal.science/hal-00834645}

Submitted on 17 Jun 2013

HAL is a multi-disciplinary open access archive for the deposit and dissemination of scientific research documents, whether they are published or not. The documents may come from teaching and research institutions in France or abroad, or from public or private research centers.
L'archive ouverte pluridisciplinaire HAL, est destinée au dépôt et à la diffusion de documents scientifiques de niveau recherche, publiés ou non, émanant des établissements d'enseignement et de recherche français ou étrangers, des laboratoires publics ou privés. 


\title{
Optimal Location of a Mobile Sensor Continuum for Environmental Monitoring
}

\author{
Didier Georges * \\ * Grenoble INP / Univ Grenoble 1 / Univ Grenoble 3 / CNRS, \\ GIPSA-lab, F-38402 Saint Martin d'Hères, \\ (e-mail: didier.georges@gipsa-lab.grenoble-inp.fr).
}

\begin{abstract}
In this paper, a nonlinear conservation law is proposed for the goal of optimal location of a mobile sensor continuum. The monitoring of pollution on a $2 \mathrm{D}$ domain is used throughout the paper to illustrate the effectiveness of the proposed approach.
\end{abstract}

Keywords: Conservation laws, sensor swarming, collective behavior, optimal sensor location.

\section{INTRODUCTION}

Research on sensors networks is a very attractive field due to the potential ability of such networks to monitor or track distributed environmental phenomena (weather, seismic events, wildfires, air, soil or river pollution ...) Nowadays monitoring systems are mostly based on static networks of sensors, Ghanem (2004). However the use of mobile sensors can potentially provide more versatility in collecting distributed information. An additional advantage of mobile sensor networks is their exploration capability in hazardous environments.

In most of the existing approaches, the navigation of mobile sensors for environmental monitoring exploits ad hoc systematic exploration techniques by using concentration gradient and flow direction to track plums to their source, Cortes (2004); Trincavelli (2008); Zarzhitsky (2005). However in the infinite-dimensional framework, we can mention a different approach in Demetriou (2011); Ucinski (2005) whose goal is to derive a stable distributed parameter state observer by using measurements from some mobile sensors which have also to be controlled to satisfy this goal.

In Georges (2013), the use of an observability index based on the so-called observability gramian of a reduced model of the PDE governing pollution is proposed. The objective is mainly to get an optimal configuration of the sensors suitable to enhance the performance of state observers designed for estimation or prediction of the distributed pollution dynamics. In Georges (2013), a navigation strategy of a mobile sensor network is proposed which maximizes the observability index while providing a optimal configuration of the sensors under sensor interdistance constraints, thanks to a gradient search. The main goal of this paper is to extend this approach by moving from individual mobile sensors (microscopic approach) to mobile sensor swarms represented by some continua (macroscopic approach) (see Ha (2008), Canizo (2012), Colombo (2012), Albi (2012)), which only requires a rather simple numerical simulation.

Even if the attention here is paid to advection-diffusion PDEs, many applications are available, which are not limited to the case of advection-diffusion PDEs: Monitoring of pollution advection-diffusion in $1 \mathrm{D}$ or $2 \mathrm{D}$ hydraulic systems coupled to Saint-Venant (shallow water) equations (rivers, lakes, estuaries or seas), underseas pollution monitoring, 2D or 3D air pollution monitoring, seismic monitoring, or monitoring of large infrastructures in civil engineering (dams for instance), for some important examples.

The organization of the paper is now as follows. In section 2 , some background is provided on advection-diffusion PDE for pollution modeling. Section 3 is devoted to the definition of an observability index derived from the notion of observability gramian. In section 4 , a nonlinear conservation law is derived for the goal of optimal sensor location. In section 5, an illustrative example is proposed. Finally the paper ends with some conclusions and perspectives.

\section{THE ADVECTION-DIFFUSION PDE FOR POLLUTION MODELING}

Pollution dispersion (without chemical reaction) may be well modeled (see Tirabassi (1989) and Zannetti (1990) for instance, in the case of air pollution) on a domain $\Omega$ by an advection-diffusion partial differential equation of the form:

$$
\frac{\partial z}{\partial t}(x, t)+U(x, t) \cdot \nabla z(x, t)=K \triangle z(x, t)+D(x, t) S(t \chi 1)
$$

where $x \in \Omega \subset \mathbb{R}^{N}$, with $N=1,2$ or $3, z(x, t)$ is the concentration of a chemical species (the pollutant), $U(x, t)$ is a vector of flow velocities which is supposed to be known (through measurements or computation of other PDEs, such as the Saint-Venant equations governing the dynamics of open-channel hydraulic systems or meteorological models in the case of air pollution), $K$ is a constant diffusion coefficient which is supposed to be known, and $D(x, t)$ is the operator defining how the source of pollutant $S(t)$ acts in the domain $\Omega . \nabla$ and $\triangle$ stand for the gradient and the Laplacian respectively. ". " denotes the standard scalar product in $\mathbb{R}^{2}$.

Some initial conditions $z(x, t=0)=z_{0}(x)$ and some boundary conditions on the domain boundary $\partial \Omega$ have to 
be provided. In this paper and without restriction, some Dirichlet boundary conditions will be considered:

$$
z(x, t)=z_{b c}(x, t), \forall x \in \partial \Omega \text {. }
$$

\section{AN OBSERVABILITY INDEX BASED ON THE OBSERVABILITY GRAMIAN}

\subsection{Some background on observability function and gramian}

For finite-dimensional systems, the so-called "transient observability function" is defined as (Brockett (1970))

$$
L_{o}(X, T)=\frac{1}{2} \int_{0}^{T}\|y(t)\|^{2} d t, x(0)=X,
$$

that is the output energy generated by any initial state $X \in R^{n}$ in the time interval $[0, T] . L_{o}$ may be rewritten as

$$
L_{o}(X, T)=\frac{1}{2} X^{T}\left(\int_{0}^{T} \Phi^{T}(t, 0) C(t)^{T} C(t) \Phi(t, 0) d t\right) X
$$

with $\frac{d \Phi(t, \tau)}{d t}=A(t) \Phi(t, \tau), \Phi(t, t)=I_{d}$, if we consider some continuous-time time-varying linear systems described in state-space form by

$$
\begin{aligned}
& \dot{x}=A(t) x \\
& y=C(t) x
\end{aligned}
$$

where $x \in R^{n}$ and $y \in R^{p}$. We assume that the system is at least detectable.

A necessary and sufficient condition for observability (resp. detectability) of the pair $(C(t), A(t))$ is that there exists $\forall t \in[0, T]$, a positive definite (resp. non negative definite) symmetric matrix $W(t)$, solution to the following differential Lyapunov equation:

$$
\begin{aligned}
-\dot{W}(t)+A^{T}(t) W(t)+W(t) A(t) & =-C^{T}(t) C(t), \\
W(0) & =0,
\end{aligned}
$$

so-called "observability gramian" matrix, such that

$$
L_{o}(X, T)=\frac{1}{2} X^{T} W(T) X .
$$

In the case of time-invariant asymptotically stable observable (resp. detectable) linear systems defined by the pair $(C, A), L_{o}$ is finite when $T \rightarrow+\infty$ and $\lim _{T \rightarrow+\infty} W(T)=$ $\bar{W} . \bar{W}$ is obtained as the unique positive definite (resp. non negative definite) solution to the Lyapunov problem:

$$
A^{T} W+W A=-C^{T} C .
$$

It follows that $W(T)$ or $\bar{W}$ can be used as a measure of the observability degree of the system, since the singular values of $W$ or $\bar{W}$ represent the sensitivity of output $y$ to each component of any initial state $x(0)=X$.

Since our goal is to maximize the observability of a physical phenomenon, we seek for maximizing a norm of the observability gramian Georges (2013); for instance, the trace of the gramian that is the sum of the singular values of the gramian:

$$
I_{o}(0, T)=\operatorname{trace}(W(T)),
$$

where $I_{o}$ will be denoted as the transient observability index, while $I_{a o}=I_{o}(0,+\infty)=\operatorname{trace}(\bar{W})$ will be denoted as the asymptotic observability index in what follows.

The extension of the gramian notion to infinite-dimensional systems has been performed (see Curtain (1995), p. 154-156) for linear infinite-dimensional operators, acting as infinitesimal generators of a $C_{0}$-semigroup. If we consider the the system defined in abstract form by

$$
\begin{gathered}
\dot{z}(t)=A z(t) \\
y(t)=C z(t),
\end{gathered}
$$

where $A$ is the infinitesimal generator of a $C_{0}$-semigroup $T(t)$ on a Hilbert space $Z$, and $C$ is an output linear and bounded operator from $Z$ to a Hilbert space $Y$, the pair $(A, C)$ is said to be approximately observable on $[0, T]$ (for some finite $T>0$ ), if the so-called observability map of $(A, C)$ on $[0, T]$, which is the bounded linear map $\mathcal{C}^{T}: Z \rightarrow L_{2}([0, T] ; Y)$ defined by

$$
\mathcal{C}^{T} z=C T(.) z
$$

is such that $\operatorname{ker} \mathcal{C}^{T}=\{0\}$. Furthermore the observability gramian of $(A, C)$, which is defined by the following linear self-adjoint operator

$$
W^{T}=\mathcal{C}^{*} \mathcal{C}
$$

is such that $\mathcal{W}^{T}>0$.

Similarly for exponentially stable systems and for $T \rightarrow$ $+\infty$, the observability gramian $W^{\infty}$ is the unique selfadjoint solution to the Lyapunov operator equation for any $z_{1}, z_{2} \in D\left(A^{*}\right)$

$$
<W^{\infty} z_{1}, A z_{2}>+<A z_{1}, W^{\infty} z_{2}>=-<C z_{1}, C z_{2}>,
$$

where $<., .>$ is the associated inner product.

Finding an explicit solution to this Lyapunov equation is not possible in general, except for Riesz-spectral operators, whose eigenvalues and eigenfunctions are known explicitely (for instance, the heat equation, see Liu (2010)). In Vaidya (2011), an approximate solution $W^{T}$ is performed in the case of an advection PDE. In this paper, we will not follow this way. In order to derive the observability index for our advection-diffusion problem, an approximate finite-dimensional model is proposed in the next section, thanks to a Galerkin method, before deriving the hereproposed observability index on the basis of this projected model.

\subsection{A reduced-order model based on a Galerkin method}

As in Georges (2013), a Galerkin weighted-residual method (known for providing accurate solutions, see Fletcher (1984)) is proposed with the goal of deriving a reducedorder finite-dimensional model of the ADPDE, which does not rely on a spatial grid, while ensuring some regularity properties. 
It consists in seeking an approximate $z_{a}(x, t)$ of (1)-(2) as a linear combination of $N$ basis functions $\phi_{i}(x), i=1, \ldots, N$ in a $N$-dimensional subspace:

$$
z_{a}(x, t)=\sum_{i=1}^{N} z_{i}(t) \phi_{i}(x),
$$

where the $z_{i}(t)$ 's are the coordinates in the function basis.

The problem consists now in introducing (16) in both (1) and (2) to derive two residuals:

$$
\begin{aligned}
R(x, t) & =\frac{\partial z_{a}}{\partial t}(x, t)+U(x, t) \cdot \nabla z_{a}(x, t) \\
& -K \triangle z_{a}(x, t)-D(x, t) S(t), \forall x \in \Omega, \\
\bar{R}(x, t) & =z_{a}(x, t)-z_{b c}(x, t), \forall x \in \partial \Omega .
\end{aligned}
$$

The problem is to render these residuals smaller as possible (ideally equal to zero). In order to get this result, a solution will consist in computing $z_{a}(x, t)$ such that both $R$ and $\bar{R}$ are orthogonal to each basis function $\phi_{i}(x)$ :

$$
\begin{aligned}
\int_{\Omega} R(x, t) \phi_{j}(x) d \Omega & =0, j=1, \ldots, N \\
\int_{\partial \Omega} \bar{R}(x, t) \phi_{j}(x) d \partial \Omega & =0 .
\end{aligned}
$$

These orthogonality conditions can be also simplified as:

$$
\begin{array}{r}
\int_{\Omega} R(x, t) \phi_{j}(x) d \Omega+\gamma \int_{\partial \Omega} \bar{R}(x, t) \phi_{j}(x) d \partial \Omega=0, \\
j=1, \ldots, N,
\end{array}
$$

where $\gamma$ is a real weighting coefficient non equal to zero.

As in Georges (2013)) we used a function basis obtained apart from Legendre's orthogonal polynomials. In the case of $1 \mathrm{D}$ problems defined on a domain $\Omega=[-1,+1]$, Legendre's polynomials are given by the sequence $\left\{L_{i}(x)\right\}_{i \geq 0}$

$$
\begin{array}{r}
(i+1) L_{i+1}(x)=(2 i+1) x L_{i}(x)-i L_{i-1}(x), \\
L_{0}(x)=1, L_{1}(x)=x,
\end{array}
$$

which are orthogonal on $[-1,+1]$ with unitary weight:

$$
\int_{-1}^{1} L_{i}(x) L_{j}(x) d x=0, \forall i, j, i \neq j
$$

and

$$
\int_{-1}^{1} L_{i}(x) L_{i}(x) d x=\frac{2}{2 i+1}, \forall i \geq 0 .
$$

In the $2 \mathrm{D}$ case (using spatial coordinates $x=\left(x_{1}, x_{2}\right)$ ), we get

$$
\begin{array}{r}
\phi_{k}\left(x_{1}, x_{2}\right)=L_{i}\left(x_{1}\right) \times L_{j}\left(x_{2}\right), k=i+(j-1) N, \\
i, j=1, \ldots, N .
\end{array}
$$

Using this function basis leads to a linear dynamical system expressed in the coordinates $z_{a}(t)=\left(z_{a}^{1}(t), \ldots, z_{a}^{N}(t)\right)^{T}$ :

$$
E \dot{z}_{a}(t)=A(t) z_{a}(t)+B(t) S(t)+F(t), z_{a}(0)=z_{a}^{0},(26)
$$
where the matrices $E$ (which is diagonal, thanks to basis orthogonality), $A$ and the vectors $B$ and $F$ have elements defined as follows:

$$
E_{i i}=\int_{\Omega} \phi_{i}(x) \phi_{i}(x) d \Omega
$$

$$
E_{i j}=\int_{\Omega} \phi_{i}(x) \phi_{j}(x) d \Omega=0, \forall i, j, i \neq j
$$

$$
\begin{gathered}
A_{i j}(t)=\int_{\Omega}\left[-U(x, t) \cdot \nabla \phi_{i}(x)+K \triangle \phi_{i}(x)\right] \phi_{j}(x) d \Omega \\
+\gamma \int_{\partial \Omega} \phi_{i}(x) \phi_{j}(x) d \partial \Omega(29 \\
B_{j}(t)=\int_{\Omega} D(x, t) \phi_{j}(x) d \Omega
\end{gathered}
$$

and

$$
F_{j}(t)=-\gamma \int_{\partial \Omega} z_{b c}(x, t) \phi_{j}(x) d \partial \Omega
$$

and where the initial state vector $z_{a}^{0}$ is computed as the solution of the linear system

$$
\begin{array}{r}
\int_{\Omega}\left[z_{a}(x, 0)-z_{0}(x)\right] \phi_{j}(x) d \Omega \\
+\gamma \int_{\partial \Omega}\left[z_{a}(x, 0)-z_{b c}(x, 0)\right] \phi_{j}(x) d \partial \Omega=0, \\
j=1, \ldots, N .
\end{array}
$$

which is equivalent to

$$
G z_{a}^{0}+H=0
$$

where $G$ and $H$ are defined by

$$
G_{i j}=\int_{\Omega} \phi_{i}(x) \phi_{j}(x) d \Omega+\gamma \int_{\partial \Omega} \phi_{i}(x) \phi_{j}(x) d \partial \Omega
$$

and

$$
H_{j}=-\int_{\Omega} z_{0}(x) \phi_{j}(x) d \Omega-\gamma \int_{\partial \Omega} z_{b c}(x, 0) \phi_{j}(x) d \partial \Omega
$$

3.3 An observability index for the reduced model of the $A D P D E$

If $M$ sensors are placed at position $x_{i} \in \Omega, i=1, \ldots, M$ the resulting output energy on $[0, T]$ will be defined, thanks to reduced model (26), by

$$
\begin{aligned}
L_{o}\left(Z_{a}\right) & =\frac{1}{2} Z_{a}^{T}\left\{\int_{0}^{T} \phi^{T}(t, 0) \sum_{i=1}^{M} C^{T}\left(x_{i}\right) C\left(x_{i}\right) \phi(t, 0) d t\right\} Z_{a} \\
& =\frac{1}{2} Z_{A}^{T} W\left(x_{1}, \ldots, x_{M}, T\right) Z_{A} \\
& =\frac{1}{2} Z_{a}^{T} \sum_{i=1}^{M}\left\{\int_{0}^{T} \phi^{T}(t, 0) C^{T}\left(x_{i}\right) C\left(x_{i}\right) \phi(t, 0) d t\right\} Z_{a} \\
& =\frac{1}{2} Z_{A}^{T} \sum_{i=1}^{M} W_{s}\left(x_{i}, T\right) Z_{A}
\end{aligned}
$$

where $Z_{a}$ is the initial state generating the output on $[0, T], \phi(.,$.$) is defined by \frac{d \Phi(t, \tau)}{d t}=E^{-1} A(t) \Phi(t, \tau)$, $\Phi(t, t)=I_{d}, C(x)$ is defined by 


$$
C(x)=\left(\phi_{1}(x) \ldots \phi_{N}(x)\right),
$$

$W\left(x_{1}, \ldots, x_{M}\right)$ is the global observability gramian, while $W_{s}\left(x_{i}, T\right)$ is the elementary observability gramian associated to sensor $i$.

Now we use the following assumption in what follows:

Assumption: the time-varying velocity field $U(x, t)$ is replaced by a mean velocity field defined over a finite time interval $[0, T]$

$$
U(x)=\frac{1}{T} \int_{0}^{T} U(x, t) d t .
$$

This assumption means that the velocity field $U(x, t)$ is available through measurements or computation over $[0, T]$.

By using this assumption, matrix $A$ of (26) is no more time-varying and the observability index $I_{a o}$ can be computed by considering any fixed configuration $\left(x_{1}, \ldots, x_{M}\right)$ of the $M$ sensors in domain $\Omega . I_{a o}=\operatorname{trace}\left(\bar{W}\left(x_{1}, \ldots, x_{M}\right)\right)$ where $\bar{W}\left(x_{1}, \ldots, x_{M}\right)$ is computed as solution to the following Lyapunov equation:

$$
H^{T} W+W H=-\sum_{i=1}^{M} C^{T}\left(x_{i}\right) C\left(x_{i}\right),
$$

where $H=E^{-1} A$ is an Hurwitz matrix and $I_{a o}\left(x_{1}, \ldots, x_{M}\right)=$ $\operatorname{trace}\left(\bar{W}\left(x_{1}, \ldots, x_{M}\right)\right)$.

\section{A CONSERVATION LAW FOR THE OPTIMAL LOCATION OF A SENSOR CONTINUUM}

\subsection{A microscopic approach}

The navigation of $M$ mobile robots using a potential approach is well known in the literature, see Gazi (2007), Kowalczyk (2005) for instance. The common goal of all the sensors is therefore to reach an optimal configuration $x_{i}^{*}$, $i=1, \ldots, M$ such that the gradient of the potential $P$ is equal to zero, that is a solution to the necessary conditions of the optimization problem

$$
\min _{x_{i}, i=1, \ldots, M} P\left(x_{1}, \ldots, x_{M}\right) .
$$

In our case, the choice of the functional $P$ is naturally given by

$$
P\left(x_{1}, \ldots, x_{M}\right)=-I_{a o}\left(x_{1}, \ldots, x_{M}\right)
$$

since the goal is to find an optimal configuration which maximizes the observability index.

Finally, without robot interdistance constraints, the trajectory $x_{i}^{k}$ of each sensor $i$ is obtained from the following gradient descent method:

$$
x_{i}^{k+1}=x_{i}^{k}+\delta \nabla_{x_{i}} I_{a o}\left(x_{1}^{k}, \ldots, x_{M}^{k}\right), i=1, \ldots, M
$$

The navigation will end when $\left\|x^{k+1}-x^{k}\right\|<\epsilon_{s}$, where $\epsilon_{s}$ is a given reached precision and $\delta>0$ is small enough to ensure convergence of the sequence $x_{i}^{k}, i=1, \ldots, M$. If the convergence is reached, then the cluster point satisfies the necessary optimality conditions $\nabla_{x_{i}} I_{a o}=0$. The gradient $\nabla_{x_{i}} I_{a o}\left(x_{1}, \ldots, x_{M}\right)$ is defined as $\nabla_{x} \operatorname{trace}\left(\bar{W}\left(x_{1}, \ldots, x_{M}\right)\right)$. This "microscopic" approach has been successfully applied in Georges (2013). Its main drawback is obviously computational complexity when the number of mobile sensors increases.

\subsection{A macroscopic approach}

Let $\rho^{M}(x, t)$ denote the following empirical measure defined by

$$
\rho^{M}(x, t)=\sum_{i=1}^{M} \delta\left(x-x_{i}(t)\right) .
$$

Then, for $T \rightarrow+\infty$ and under the mean velocity field assumption, output energy (36) may be rewritten as

$$
\begin{aligned}
L_{o}\left(x,+\infty, Z_{a}\right)= & \frac{1}{2} Z_{a}^{T}\left(\int _ { \Omega } \left[\int_{0}^{\infty} e^{H^{T} t} \times\right.\right. \\
& \left.\left.C^{T}(x) C(x) e^{H t} d t\right] \rho^{M}(x, t) d \Omega\right) Z_{a} \\
= & \frac{1}{2} Z_{A}^{T}\left(\int_{\Omega} \bar{W}(x) \rho^{M}(x, t) d \Omega\right) Z_{A}
\end{aligned}
$$

where $\bar{W}(x)$ is solution to

$$
H^{T} W+W H=-C^{T}(x) C(x) .
$$

Then $I_{a o}$ may be expressed as follows

$$
I_{a o}=\int_{\Omega} \operatorname{trace}(\bar{W}(x)) \rho^{M}(x, t) d \Omega
$$

The necessary conditions for optimality (observability maximization) are recovered from

$$
\nabla_{x_{i}} I_{a o}=\nabla_{x} \operatorname{trace}\left(\bar{W}\left(x=x_{i}\right)\right) \rho^{M}\left(x=x_{i}, t\right)=0
$$

where

$$
\nabla_{x} \operatorname{trace}(\bar{W}(x))=\left(\sum_{i=1}^{N} \bar{W}_{i, i, 1}^{\prime}(x), \ldots, \sum_{i=1}^{N} \bar{W}_{i, i, \operatorname{dim}(\Omega)}^{\prime}(x)\right)^{T}
$$

and tensor $\left.\bar{W}^{\prime}(x)\right)$ is a third order tensor, solution to the following tensorial equation

$$
\begin{aligned}
H^{T} \otimes \bar{W}^{\prime}+\bar{W}^{\prime} \otimes H= & -C^{T}(x) \nabla_{x} C(x) \\
& -\nabla_{x} C^{T}(x) C(x) .
\end{aligned}
$$

Now let us denote $\dot{x}_{i}(t) \approx \frac{\left(x_{i}^{k+1}-x_{i}^{k}\right)}{\delta}$. Equation (42) may be recovered as follows

$$
\dot{x}_{i}(t)=\nabla_{x} \operatorname{trace}\left(\bar{W}\left(x=x_{i}\right)\right) .
$$

which defines the velocity of each sensor $i$.

Inspired by (49) and following the same approach as in $\mathrm{Ha}$ (2008), Canizo (2012) and Albi (2012) for crowd modeling, when $\rho^{M}(x, t) \rightarrow \rho(x, t)$ for $M \rightarrow \infty$, the following conservation law is proposed, if the conservation of sensor density $\rho(x, t)$ w.r.t. time is assumed.

$$
\partial_{t} \rho(x, t)+\operatorname{div}_{x}(\rho(x, t) V(\rho(x, t), x))=0,
$$

with 
$V(\rho(x, t), x)=\delta\left(1-\rho(x, t) / \rho_{\max }\right) \nabla_{x} \operatorname{trace}(\bar{W}(x)) .(51)$ Mainly under the rather mild assumption $\nabla_{x} \operatorname{trace}(\bar{W}(x))$ is smooth, the existence and uniqueness of the solution in $L^{1}\left(\mathbb{R}^{2},\left[0, \rho_{\max }\right]\right)$ to Cauchy problem

$$
\begin{array}{r}
\partial_{t} \rho(x, t)+\operatorname{div}_{x}(\rho(x, t) V(\rho(x, t), x))=0, \\
\rho(x, 0)=\rho_{0}(x)
\end{array}
$$

is ensured (see Colombo (2012)).

\subsection{Obstacle avoidance}

In practice, obstacles should be often taken into account. For that purpose, the velocity field may be modified by adding a repulsive gradient $G_{i}$ around each obstacle $i$, $i=1, \ldots, N_{o}$ :

$$
G_{i}(x)=\alpha_{i}\left(x-x_{i}^{o}\right) e^{-\beta_{i}\left\|x-x_{i}^{o}\right\|},
$$

where $x_{i}^{o}$ denotes the coordinates of obstacle $i$, and $\alpha_{i}, \beta_{i}$ are some positive coefficient. The velocity field becomes

$$
\begin{aligned}
V(\rho(x, t), x) & =\delta\left(1-\rho(x, t) / \rho_{\max }\right)\left\{\nabla_{x} \operatorname{trace}(\bar{W}(x))\right. \\
& \left.+\sum_{i=1}^{N_{o}} G_{i}(x)\right\} .
\end{aligned}
$$

Again this problem has been studied in Colombo (2012) (Nonlocal route choice) where existence and unicity of the solution $L^{1}\left(\mathbb{R}^{2},\left[0, \rho_{\max }\right]\right)$ holds under additional assumption that the $G_{i}$ 's are smooth.

\subsection{Asymptotic collective behavior}

The stable equilibrium behavior $\rho_{e}(x)$ is given by

$$
\begin{aligned}
V\left(\rho_{e}(x, t), x\right) & =\delta\left(1-\rho_{e}(x, t) / \rho_{\max }\right)\left\{\nabla_{x} \operatorname{trace}(\bar{W}(x))\right. \\
& \left.+\sum_{i=1}^{N_{o}} G_{i}(x)\right\}=0 .
\end{aligned}
$$

$\nabla_{x} \operatorname{trace}(\bar{W}(x))+\sum_{i=1}^{N_{o}} G_{i}(x)=0$ corresponds to the optimality condition for observability maximization or for the trade-off between observability maximization and obstacle avoidance in the presence of obstacles.

\section{A NUMERICAL EXAMPLE}

Now we consider the optimal navigation of a sensor continuum towards the equilibrium configuration satisfying necessary conditions, on domain $\Omega=[0 \mathrm{~km}, 1 \mathrm{~km}]^{2}$. The ADPDE parameters used in the simulation are given in table 1 , where the velocity field $U=\left(U_{x}, U_{y}\right)$ is supposed to be uniform over the domain and constant with respect to time. The reduced model of the ADPDE is obtained by using 20 Legendre polynomials in each spatial direction as basis functions and with $\gamma=-1 e^{4}$.

The simulations was performed by using a $2 \mathrm{D}$ LaxFriedrichs integration scheme and compared to a B2Q9 Lattice Bolzmann method (see Wolfram (1986), which provides here more accurate results with more computational efforts). Two cases are considered: navigation with and without obstacles on the path. The initial density is given by $\rho_{0}(x)=e^{\left\|x-x_{I}\right\|^{2} / 0.005}$, with $x_{I}=(0.3,0.3)$. We consider three fixed obstacles placed at $(0.3,0.4),(0.4,0.3)$ and $(0.3,0.3)$, with $\alpha_{i}=120$ and $\beta_{i}=40$. The other coefficients are $\delta=2$ and $\rho_{\max }=1$. The results for the case without obstacles are given by Fig. 1-3, while the results with obstacles are given by Fig. 4-6. The fixed level curves on the upper right part of the figures corresponds to the pollution density levels at equilibrium. The results compare well to those obtained in Georges (2013) with the microscopic approach.

Table 1. ADPDE parameters

\begin{tabular}{|c||c||c||c||c|}
\hline$U_{x}$ & $U_{y}$ & $K$ & B.C. & $D(x, t)$ \\
\hline $10 \mathrm{~km} / \mathrm{h}$ & $10 \mathrm{~km} / \mathrm{h}$ & 1 & $z_{b c}(x, t)=0$ & $e^{-\frac{\|x\|^{2}}{2 \sigma^{2}}}, \sigma=0.01$ \\
\hline
\end{tabular}

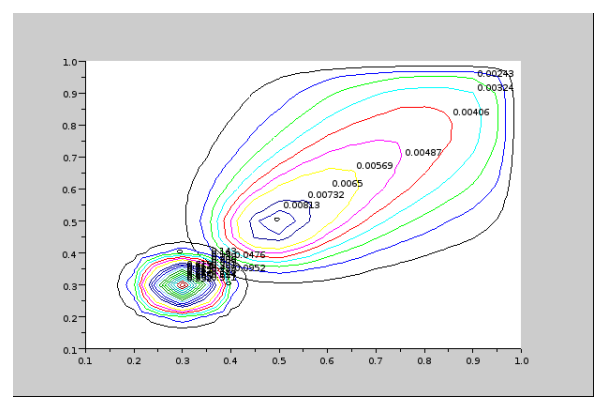

Fig. 1. Initial configuration $(t=0)$ - without obstacles.

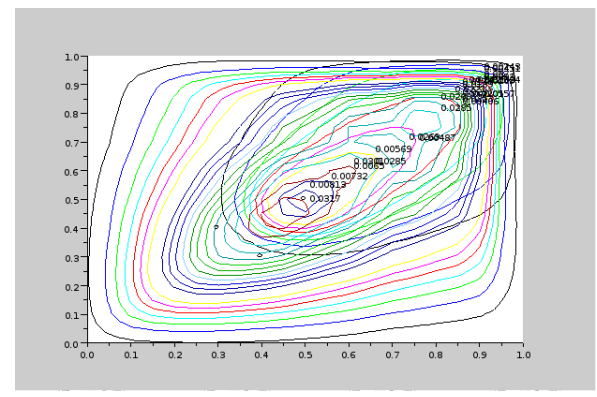

Fig. 2. Sensor density $(t=4)$ - without obstacles.

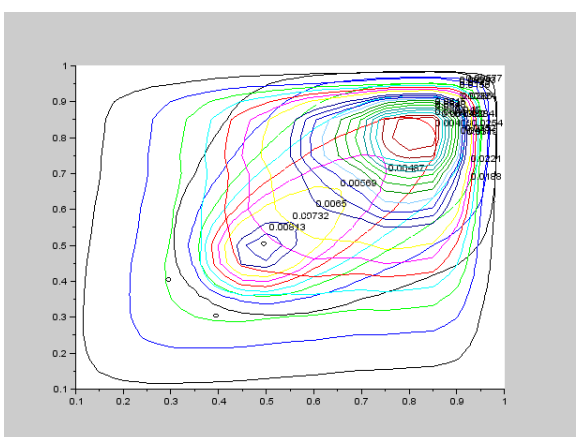

Fig. 3. Sensor density $(t=8)$ - without obstacles.

\section{CONCLUSIONS AND PERSPECTIVES}

A nonlinear conservation law is proposed to model the collective behavior of a mobile sensor continuum used for monitoring purpose. The sensor density reaches an 


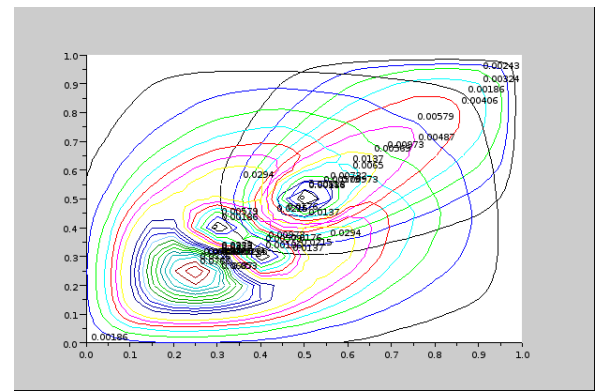

Fig. 4. Sensor density $(t=2)$ - with obstacles.

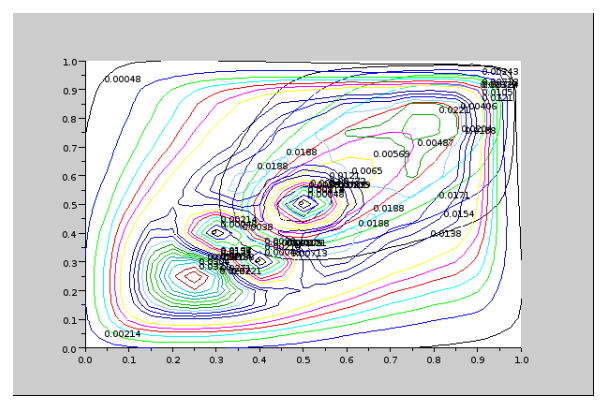

Fig. 5. Sensor density $(t=4)$ - with obstacles.

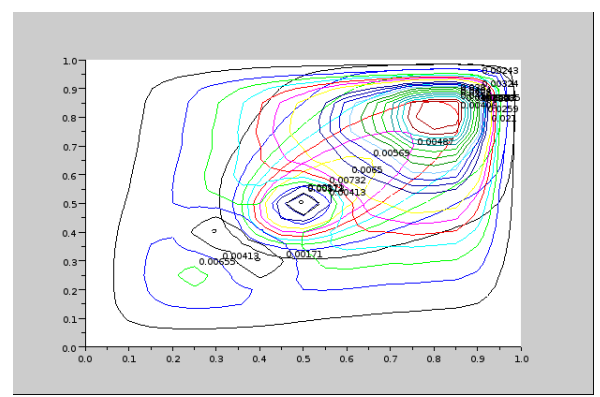

Fig. 6. Sensor density $(t=8)$ - with obstacles.

equilibrium corresponding to the necessary conditions for optimality (observability maximization or trade-off between observability maximization and obstacle avoidance in the presence of obstacles). Future works will be devoted to a conservation law derivation based on the infinitedimensional observability gramian and the coupling with infinite-dimensional observer design.

\section{REFERENCES}

Albi, G., and Pareschi, L. (2012). Modeling Self-Organized Systems Interacting with few Individuals: from Microscopic to Macroscopic Dynamics. Applied Math. Letters, http://dx.doi.org/10.1016/j.aml.2012.10.011.

Brockett, R.W. (1970). Finite dimensional linear systems. New York: Wiley, 1970.

Canizo, J.A., Carrillo, J.A., Rosado J. (2012). A wellposedness theory in measures for some kinetic models of collective motion. Math. Models Methods Appl. Sci. 21 (2011) 515539.

Colombo, R.M., Garavello, M., Lécureux-Mercier, M. and Pogodaev, N. (2012). Conservation Laws in the Modeling of Moving Crowds. In Proceedings of HYP2012, Padova, Italy.
Cortes, J., Martinez, S., Karatas, T., and Bullo, F. (2004). Coverage control for mobile sensing networks. IEEE Transactions on Robotics and Automation, Volume: 20 Issue: 2, April 2004.

Curtain, R.F., and Zwart, H.J. (1995). An Introduction to Infinite-Dimensional Linear Systems Theory. SpringerVerlag, ISBN 0-387-94475-3.

Demetriou, M.A., and Ucinski, D. (2011). State estimation of spatially distributed processes using mobile sensing agents. Proceedings of the American Control Conference, 2011.

Fletcher C.A.J. (1984). Computational Galerkin methods, Springer Series in Computational Physics. SpringerVerlag, 1984.

Gazi, V., and Fidan, B. (2007). Coordination and control of multi-agent dynamic systems: Models and approaches. Lecture notes in Computer Science, Springer, 2007.

Georges, D. (2013). Optimal Location of Mobile Sensors for Environmental Monitoring. Proceedings of the European Control Conference, Zurich, Switzerland, july 2013.

Ghanem, M., Guo Y., Hassard, J., Osmond, M., Richards, M. (2004). Sensor Grids For Air Pollution Monitoring. In Proc. 3rd UK e-Science All Hands Meeting, 2004.

Ha, S., and Tadmor, E. (2008). From particle to kinetic and hydrodynamic descriptions of flocking. Kinet. Relat. Models 1 (2008) 415 - 435.

Kowalczyk, W., and Koslovski, K. (2005). Artificial potential based control for a large-scale formation of robots. Climbing and walking robots, Part. II, pp. 191199, Springer, 2005.

Liu, W. (2010). Elementary feedback Stabilization of Linear reaction-Convection-Diffusion Equation and the Wave Equation. Springer-Verlag, DOI 10.1007/978-3$642-04613-1 \_4$.

Tirabassi, T. (1989). Analytical air pollution advection and diffusion models. Water, Air, \& Soil Pollution, Volume 47, Numbers 1-2, september 1989.

Trincavelli, M., Reggente, M.,Coradeschi, S., Loutfi, A., Ishida, H., Lilienthal A.J. (2008). Towards environmental Monitoring with mobile robots. Proceedings of the IEEE/RSJ International Conference on Intelligent Robots and Systems, 2008.

Ucinski, D. (2005). Optimal Measurement Methods for Distributed Parameter System Identification. CRC Press, Boca Raton, 2005, ISBN: 0-8493-2313-4.

Vaidya, U., Rajarm, R., and Dasgupta, S. (2011). Actuator and sensor placement in linear advection PDE. Proceedings of the 50th CDC-ECC, Orlando, USA, 2011.

Wolfram, S. (1986). Cellular automaton fluids 1: Basic theory. J. Stat. Phys. 45:471-526.

Zannetti, P. (1990). Air Pollution Modelling. Theories, Computational Methods and Available Software. Computational Mechanics Publications, Southampton, Boston, Van Nostrand Reinhold, New York, 1990.

Zarzhitsky, D., Spears, D. F., and Spears W. (2005). Distributed Robotics Approach to Chemical Plume Tracing. Proceedings of the IEEE/RSJ International Conference on Intelligent Robots and Systems, 2005. 